\title{
INITIAL REPORT ON SPANISH PEDIATRIC ONCOLOGIC, HEMATOLOGIC AND POST STEM CELL TRANSPLANTATION PATIENTS DURING SARS-COV-2 PANDEMIC
}

Anna Faura Morros ${ }^{1}$, Susana Rives Solà ${ }^{2}$, ALVARO LASSALETTA $^{3}$, Elena Sebastián ${ }^{4}$, Luis Madero ${ }^{3}$, Jorge Huerta Aragonés ${ }^{5}$, Marina García-Morin ${ }^{6}$, Antonio Perez Martinez ${ }^{7}$, Luisa Sisinni $^{7}$, Itziar Astigarraga ${ }^{8}$, Pablo Velasco ${ }^{9}$, Luis Gros ${ }^{9}$, Lucas Moreno ${ }^{10}$, Ana Carboné $^{11}$, Carmen Rodriguez-Vigil ${ }^{11}$, Susana Riesco ${ }^{12}$, Maria del Carmen Mendoza Sanchez ${ }^{12}$, Elena Garcia Macias ${ }^{13}$, Maria Trabazo ${ }^{14}$, Montserrat Torrent ${ }^{14}$, Isabel Badell ${ }^{14}$, José Luis Fuster Soler ${ }^{15}$, Nerea Domínguez-Pinilla ${ }^{16}$, Antonio Juan Ribelles ${ }^{17}$, Vanesa Pérez-Alonso $^{18}$, Manuel Fernández Sanmartin ${ }^{19}$, Marta Baragaño ${ }^{20}$, Maite Gorostegui ${ }^{2}$, Sara Perez-Jaume ${ }^{2}$, Ana Fernández-Teijeiro ${ }^{21}$, Andres Morales La Madrid ${ }^{1}$, and Jose Luis Dapena $^{1}$

${ }^{1}$ Hospital Sant Joan de Déu

${ }^{2}$ Hospital Sant Joan de Deu

${ }^{3}$ Hospital Infantil Universitario Niño Jesús

${ }^{4}$ Hospital Infantil Universitario Nino Jesus

${ }^{5}$ Hospital General Universitario Gregorio Marañón

${ }^{6}$ Hospital General Universitario Gregorio Maranon

${ }^{7}$ Hospital Universitario La Paz

${ }^{8}$ Hospital Universitario Cruces, Biocruces Bizkaia Health Research Institute, UPV/EHU

${ }^{9}$ Hospital Universitari Vall d'Hebron

${ }^{10}$ Vall d'Hebron Hospital

${ }^{11}$ Hospital Universitario Miguel Servet

${ }^{12}$ Hospital Clínico de Salamanca

${ }^{13}$ Hospital Universitario Son Espases

${ }^{14}$ Hospital de la Santa Creu i Sant Pau

${ }^{15}$ Hospital Clinico Universitario Virgen de la Arrixaca

${ }^{16}$ Hospital Virgen de la Salud

${ }^{17}$ Hospital La Fe

${ }^{18}$ Hospital Universitario 12 de Octubre

${ }^{19}$ Hospital Clinico Universitario de Santiago de Compostela

${ }^{20}$ Hospital Quirón Madrid

${ }^{21}$ Complejo Hospitalario Virgen de la Macarena

June 8, 2020 


\begin{abstract}
Background: Since the beginning of SARS-CoV-2 pandemic, it has been widely recognized that children and adolescents seem to have milder clinical courses as compared to adult counterparts. However, there is concern that vulnerable collectives including pediatric patients treated for cancer or under immunosuppression may be at higher risk. Methods: We retrospectively collected Spanish COVID-19 cases in children and adolescents with solid and hematological malignancies, non-malignant chronic hematologic conditions, and post allogeneic-stem cell transplantation, from the beginning of the pandemic on January 31 to April 24, 2020. Results: We included 47 cases with RT-PCR positive COVID-19 from 41 centers in Spain, where 97.6\% of pediatric patients are treated for cancer. In most cases $(76.6 \%)$, infection was asymptomatic, or symptoms were mild. Severe illness was observed in $14.9 \%$ of cases with respiratory distress and/or hypoxemia, and $8.5 \%$ required admission to the PICU. Symptomatic patients received supportive care associated with antiviral and immunomodulatory agents depending upon severity. Anticancer therapy was withhold in the majority of cases during the infection course. Most patients recovered from COVID-19. Two deaths were reported. Conclusion: In our cohort, most children receiving anticancer chemotherapy presented a mild clinical course and had a good outcome. Highly immunosuppressed patients with major comorbidities were at higher risk of severe infections. Among this fragile collective, individualized expert discussion is critical for anti-infectious therapy and appropriate anticancer treatment.
\end{abstract}

INITIAL REPORT ON SPANISH PEDIATRIC ONCOLOGIC, HEMATOLOGIC AND POST STEM CELL TRANSPLANTATION PATIENTS DURING SARS-COV-2 PANDEMIC

\author{
A short running title: Spanish immunosuppressed children and SARS-COV-2
}

Faura $\mathrm{A}^{1}$. Rives $\mathrm{S}^{1}$. Lassaletta $\mathrm{A}^{2}$. Sebastián $\mathrm{E}^{2}$. Madero $\mathrm{L}^{2}$. Huerta $\mathrm{J}^{3}$. García-Morín $\mathrm{M}^{3}$. Pérez $\mathrm{A}^{4}$. Sisinni $\mathrm{L}^{4}$. Astigarraga $\mathrm{I}^{5}$. Velasco $\mathrm{P}^{6}$. Gros $\mathrm{L}^{6}$. Moreno $\mathrm{L}^{6}$ Carboné $\mathrm{A}^{7}$. Rodríguez-Vigil $\mathrm{C}^{7}$. Riesco $\mathrm{S}^{8}$. Mendoza $\mathrm{MC}^{8}$. García $\mathrm{E}^{9}$. Trabazo $\mathrm{M}^{10}$. Torrent $\mathrm{M}^{10}$. Badell $\mathrm{I}^{10}$. Fuster $\mathrm{JL}^{11}$. Dominguez $\mathrm{N}^{12}$. Juan Ribelles $\mathrm{A}^{13}$. Pérez $\mathrm{V}^{14}$. Fernández Sanmartín $\mathrm{M}^{15}$ Baragaño $\mathrm{M}^{16}$. Gorostegui $\mathrm{M}^{1}$. Perez-Jaume $\mathrm{S}^{1,18}$. Fernández-Teijeiro $\mathrm{A}^{16}$. Morales La Madrid $\mathrm{A}^{1^{*}}$. Dapena $\mathrm{JL}^{1}$.

1. Pediatric Oncology and Hematology Department. Hospital Sant Joan de Deu, Spain.

2. Pediatric Hematology-Oncology Department. Hospital Universitario Niño Jesús. Madrid. Spain

3. Pediatric Hematology-Oncology Department. Hospital Materno-Infantil Gregorio Marañón. Madrid. Spain

4. Pediatric Hemato-Oncology Department. Hospital Universitario La Paz. Madrid. Spain

5. Department of Pediatrics, BioCruces Bizkaia Health Research Institute, Hospital Universitario Cruces, University of the Basque Country UPV/EHU, Barakaldo, Spain.

6. Pediatric Oncology and Hematology. Vall d'Hebron Barcelona Hospital Campus. Spain

7. Pediatric Hemato-Oncology Department. Hospital Universitario Miguel Servet. Zaragoza. Spain

8. Pediatric Hemato-Oncology Department. Hospital Clínico Universitario. Salamanca. Spain

9. Pediatric Hemato-Oncology Department. Hospital Universitario Son Espases. Mallorca. Spain

10. Pediatric Oncology and Hematology Department. Hospital de la Santa Creu i Sant Pau. Barcelona. Spain.

11. Pediatric Oncology and Hematology Department. Hospital Clínico Universitario Virgen de la Arrixaca, Instituto Murciano de Investigación Biosanitaria (IMIB). Murcia. Spain

12. Pediatric Oncology and Hematology Department. Hospital Virgen de la Salud. Toledo. Spain.

13. Pediatric Oncology and Hematology Unit. Hospital Universitario La Fe. La Valencia, Spain

14. Pediatric Oncology and Hematology Department. Hospital Universitario 12 de Octubre. Madrid. Spain.

15. Pediatric Oncology and Hematology Department. Hospital Clinico Universitario de Santiago de Compostela. Spain

16. Pediatric Oncology and Hematology Department. Hospital Universitario Quiron Salud. Madrid. Spain

17. Pediatric Oncology Unit, Hospitales Universitarios Virgen Macarena y Virgen del Rocio, Sevilla, Spain.

18. Developmental Tumor Biology Laboratory, Institut de Recerca Sant Joan de Déu, Esplugues de Llobregat, Spain

*Corresponding author: 
Andres Morales La Madrid, MD MHM

Pediatric Neuro-Oncology, Department of Pediatric Hematology and Oncology

Hospital Sant Joan de Deu

Passeig Sant Joan de Deu 2, 08950. Barcelona, Spain

Email: amorales@sjdhospitalbarcelona.org

Text word count: 2493

Abstract word count: 224

Brief running title: COVID-19 in oncologic children with immunosuppression

Keywords: COVID-19, SARS-CoV-2, pandemic, children, cancer and immunosuppression

Tables: 1

Figures: 0

Legends: 1

\begin{tabular}{ll}
\hline Abbreviations & \\
\hline B-ALL & Precursor B-cell acute lymphoblastic leukemia \\
CAR-T & Chimeric Antigen Receptor T-Cell \\
COVID-19 & Coronavirus disease 2019 \\
CT & Computerized tomography \\
HCoV & Human coronavirus \\
HLH & Hemophagocytic lymphohistiocytosis \\
ICU & Intensive Care Unit \\
PICU & Pediatric Intensive Care Unit \\
GVHD & Graft-versus-host disease \\
MAS & Macrophage activation syndrome \\
NR & Normal range \\
PHO & Pediatric hemato-oncology \\
RETI-SEHOP & Registro Español de Tumores Infantiles- Sociedad Española de Hematología y Oncología \\
RT-PCR & Reverse transcription polymerase chain reaction \\
SARS-CoV-2 & Severe acute respiratory syndrome coronavirus 2 \\
SCT & Stem-cell transplantation \\
SEHOP & Sociedad Española de Hematología y Oncologia \\
ECMO & ExtraCorporeal Membrane Oxygenation \\
\hline
\end{tabular}

Background : Since the beginning of SARS-CoV-2 pandemic, it has been widely recognized that children and adolescents seem to have milder clinical courses as compared to adult counterparts. However, there is concern that vulnerable collectives including pediatric patients treated for cancer or under immunosuppression may be at higher risk.

Methods : We retrospectively collected Spanish COVID-19 cases in children and adolescents with solid and hematological malignancies, non-malignant chronic hematologic conditions, and post allogeneic-stem cell transplantation, from the beginning of the pandemic on January 31 to April 24, 2020.

Results : We included 47 cases with RT-PCR positive COVID-19 from 41 centers in Spain, where $97.6 \%$ of pediatric patients are treated for cancer. In most cases (76.6\%), infection was asymptomatic, or symptoms were mild. Severe illness was observed in $14.9 \%$ of cases with respiratory distress and/or hypoxemia, and $8.5 \%$ required admission to the PICU. Symptomatic patients received supportive care associated with antiviral 
and immunomodulatory agents depending upon severity. Anticancer therapy was withhold in the majority of cases during the infection course. Most patients recovered from COVID-19. Two deaths were reported.

Conclusion : In our cohort, most children receiving anticancer chemotherapy presented a mild clinical course and had a good outcome. Highly immunosuppressed patients with major comorbidities were at higher risk of severe infections. Among this fragile collective, individualized expert discussion is critical for anti-infectious therapy and appropriate anticancer treatment.

\section{Background}

In December 2019, the new coronavirus SARS-CoV-2 outbreak started in Wuhan, China and rapidly progressed into a pandemic ${ }^{1}$. Europe was its next stop and Spain (first case reported on January 31, 2020) was one of the first countries registering alarming increasing numbers of both confirmed cases and deaths. As of April 24, 2020, while our health care system was struggling to expand fully, mostly with adult wards and intensive care unit (ICU) beds, 202990 cases had been tested positive and 22524 deaths were reported in the country due to COVID-19.

As widely reported, the clinical presentation of COVID-19 is heterogeneous, ranging from asymptomatic or pauci-symptomatic cases to severe forms, with an overall small percentage of patients developing respiratory insufficiency and requiring admission in the ICU. The severe forms were observed more frequently in male adults older than 65 years with certain comorbidities like diabetes, hypertension, cardiac and pulmonary conditions, and obesity ${ }^{2}$. In contrast, a number of reported series show that pediatric patients rarely develop critical illness $^{3,4}$. The reasons underlying this significant disparity in clinical presentation and outcome is still a matter of debate. In Spain, from all SARS-CoV-2 confirmed positive cases, the pediatric population (cases under the age of 19 ) represented only $0.9 \%$. Furthermore, only $2.8 \%$ of these cases required pediatric intensive care unit (PICU) admission with a mortality rate of around $0.3 \%^{5}$. Children diagnosed with cancer receiving immunosuppressive chemotherapy and cellular therapies have typically significant abnormalities in immune function. Additionally, these abnormalities may persist for months or years and puts them at a higher risk for opportunistic viral - among other - infections. To this date, reports describing the clinical and epidemiological characteristics as well as outcomes in pediatric patients with cancer and/or under immunosuppression secondary to allogeneic-stem cell transplantation, or CART cell therapy acquiring the COVID-19 are scarce. A flash survey performed on March 16, 2020 on SARS secondary to SARS-CoV-2 infections in pediatric patients with anticancer treatment was recently published, identifying only 9 patients (among 10,000 patients at risk) from several countries in Europe who presented a mild or asymptomatic course $^{6}$. Understandably, a reasonable fear exists that SARS-CoV-2, like other coronaviruses ${ }^{7}$, could cause more severe infections in immunocompromised children, as has been observed in adults ${ }^{8}$, especially in patients with hematological cancer were the highest severity index and death rate have been published ${ }^{9}$.

In this report, we present the initial Spanish experience with COVID-19 in pediatric patients affected with cancer, hematologic conditions, and post allogeneic stem cell transplantation since the beginning of this pandemic.

\section{Patients and methods}

We surveyed all 43 Spanish institutions treating pediatric cases (1 month - 18 years of age), with solid and hematologic malignancies, non-malignant hematologic conditions (those receiving immunosuppressive treatment or bone marrow failure syndrome) and after allogeneic-stem cell transplantation, or CAR-T cell therapy who were diagnosed with COVID-19 from the start of the SARS-CoV-2 pandemic on January 31 to April 24, 2020. This is a collaborative SEHOP initiative. Demographic and clinical data were collected from medical records.

This study was approved by the Hospital Sant Joan de Déu Research Ethical Committee.

Definitions: Symptoms severity was classified using the WHO criteria ${ }^{10}$ : Asymptomatic infection, mildmoderate (presence of fever, symptoms of upper respiratory tract infection, pneumonia without the need for respiratory support), severe (dyspnea, tachypnea, blood oxygen saturation [?]93\%), critical (respiratory 
failure, septic shock, and / or multiple organ dysfunction / failure). Pneumonia was diagnosed in those cases with lung field infiltrates identified in chest imaging and was considered bilateral when $>1$ lobe on each lung were affected.

Statistical Analysis : Qualitative variables were described using absolute frequencies and percentages. Quantitative variables that can be assumed to be normally distributed were described using mean and standard deviation, whereas non-normal quantitative variables were described using the median. To compare quantitative variables among groups we used the Mann-Whitney test. All the analyses were performed using $\mathrm{R}$ version 3.6.2.

\section{Results}

Information of SARS-CoV-2 positive cases was received from 41 hospitals. According to the Spanish Pediatric Cancer Registry (RETI-SEHOP), these centers are responsible for the treatment of $97.6 \%$ of pediatric cancer patients in Spain. As to non-malignant hematological cases, no global database is available due to the heterogeneity of the different diseases, but the sample seems to be representative because these chronic diseases are treated in the same Pediatric and Oncology Units.

Demographic data, underlying diagnosis, clinical features, therapy received and outcome related to RTPCR confirmed SARS-CoV-2 (nasopharyngeal swab) infection of 47 patients are summarized inTable 1 . The median age of our cohort was 8.2 years, with male predominance $(72.3 \%)$. The majority of cases had leukemia or lymphoma (51.5\%), followed by patients with solid tumors $(29.8 \%)$ as underlying condition. Seventeen percent of the patients $(n=8)$ had undergone an allo-stem-cell transplantation (SCT); five of these secondary to hematological diseases and the rest of them due to severe primary immunodeficiencies. Six patients with severe non-oncologic hematologic conditions under immunosuppressive therapy were included in the study. Three of 47 patients had received CAR T-cell therapy as salvage regimen for relapsed B-ALL. Regarding clinical impact, the majority of patients were asymptomatic $(25.5 \%)$ or had only mild symptoms (51.1\%). Fever was the most frequent symptom at presentation (51.1\%), followed by cough and rhinorrhea (40.4\%). A minority of cases presented diarrhea (4.2\%) and only two patients presented some type of cutaneous manifestation (1 case with rash, 1 case with purpuric lesions). Radiologic abnormalities (mostly by chest-X-ray, $3 \mathrm{CT}$ scans performed in critical patients) consisting in pneumonia were observed in $32.4 \%$ of the patients. The percentage of children who required hospitalization was $76.6 \%(n=32)$. However, 12 of them were already admitted when COVID-19 diagnosis was made: One due to febrile neutropenia, five for cancer treatment, 2 post-SCT, 4 not reported). Severe illness with respiratory distress and/or hypoxemia was identified in 11 patients. Four of them, all males, evolved to critical illness with progressive respiratory failure requiring admission to the PICU ( $8.5 \%$ of all patients).

The clinical course of the 4 critically-ill COVID-19 patients is summarized below:

Case 1: A 16-year-old male undergoing myelosuppressive chemotherapy for primary mediastinal large B-cell lymphoma admitted initially for severe neutropenia and pancreatitis. He developed pneumonia requiring cannula oxygen therapy; recovering soon after. Case 2 was an 8-year-old boy admitted post-SCT due to a primary immunodeficiency with graft-versus-host disease (GVHD), who presented with bilateral pneumonia requiring mechanical ventilation and ECMO. The patient presented a torpid clinical evolution and deceased. Case 3, 18 year-old male on therapy for an Ewing-like round cell sarcoma, with initial fever and cough. Thereafter he developed respiratory worsening with new bilateral infiltrates and respiratory failure, needing high flow oxygen therapy, with a favorable clinical outcome. Case 4 was an 11-year-old boy, diagnosed with relapsed B-ALL in third complete remission after CART cell therapy followed by unrelated allo-SCT. At the time of COVID-19 diagnosis, 15 months after allo-SCT, he presented an extensive chronic GVHD and poor engraftment that required several immunosuppressors. He presented initially with fever, cough, and unilateral pneumonia. Few days later developed dyspnea and hypoxemia, requiring increasing respiratory support with non-invasive ventilation and mechanical ventilation. He also developed secondary HLH, with altered coagulation, hypertriglyceridemia, hypoalbuminemia, and ferritin levels that raised up to $124000 \mathrm{ug} / \mathrm{L}$ (NR 10-120ug/L). Moreover, D-Dimer and IL-6 raised up to $2.02 \mathrm{mg} / \mathrm{dL}(\mathrm{NR}<0,5 \mathrm{mg} / \mathrm{dl})$ and $394 \mathrm{pg} / \mathrm{mL}(\mathrm{NR}<$ 
$5 \mathrm{pg} / \mathrm{ml})$, respectively. Finally, the patient died secondary to a pulmonary hemorrhage and multiorgan failure. Regarding relevant abnormal laboratory findings, critically ill patients presented in a higher frequency with severe lymphopenia (medians: 85 vs 1000, p=0.0034) and higher ferritin levels at the onset of symptoms (medians: $6666.5 \mathrm{ng} / \mathrm{ml}$ vs $1037 \mathrm{ng} / \mathrm{ml}, \mathrm{p}=0.027$ ). The maximum ferritin level was also significantly superior in critical patients admitted to the PICU in comparison to the other cases (medians: $23077 \mathrm{ng} / \mathrm{ml}$ vs $1507.5 \mathrm{ng} / \mathrm{ml}, \mathrm{p}<0.001)$. No statistically significant differences were observed in the absolute number of neutrophils and LDH. The time from clinical onset to first negative RT-PCR results was not available for all patients, but in some of them, RT-PCR remained positive after 7 weeks. Asymptomatic/mild cases (44.6\%) did not received any therapy. Twenty-three patients received hydroxychloroquine (9 patients combined with azithromycin), followed by antivirals (19.1\%) and corticosteroids (6.4\%). Six patients received monoclonal antibodies against IL1/IL6. Anti-cancer therapy was interrupted in $57.9 \%$ of the cases. Prophylactic anticoagulation was administered to five patients (10.6\%) with no thromboembolic events diagnosed. Outcome was overall good, with most symptomatic patients recovering back to baseline clinical situation. Two deaths were reported in the post allo-SCT subgroup.

\section{Discussion}

Respiratory viral infections in immunosuppressed patients usually present a more severe clinical evolution, longer viral excretion, several pulmonary complications, and a higher mortality rate ${ }^{11}$. In the specific case of $\mathrm{HCoV}$, a study published by Ogimi et al ${ }^{12}$ observed an association between the immunocompromised state and an increased risk of serious virus-related lower respiratory tract disease. During the period of data collection for this report, Spain was the second most affected country by the SARS-CoV-2 pandemic. In our country, around 1,300 children and adolescents are diagnosed with cancer every day (155.5 new cases annually per million children aged 0-14 years, similarly to other European countries) ${ }^{13}$. This report includes almost all Spanish centers with Pediatric hemato-oncology (PHO) units, providing a significative representation of the current situation in the country from the beginning of the pandemic. Thereafter, including only cancer diagnosis we observed a COVID-19 infection rate of approximately $2.5 \%$ among this patient population over the first 3 months of the pandemic. As expected, most of the reported patients (68\%) were from the States of Madrid and Catalonia, the two most affected areas in Spain by SARS-CoV-2 ${ }^{14}$. Fifteen of the patients included in the present study were previously reported in the Madrid cohort recently published by de Rojas et $\mathrm{al}^{15}$. Initial experiences from Italy (Lombardy) ${ }^{16}$, China (Wuhan) ${ }^{17,18}$ and Europe suggest a low number and severity of COVID-19 cases in pediatric patients with oncologic, hematologic, or post allo-SCT diseases $^{6}$. However, a recently published study in France, observed a higher incidence of critical patients, as 5 out of the 33 included patients were admitted to the PICU ${ }^{19}$. In addition, as the pandemic develops, and with a higher availability for viral testing, our study shows that we may expect an increasing number of reported cases in the literature. Our series represents a good initial "snapshot" of the Spanish number of cases and severity in the midst of the peak of SARS-CoV-2 cases within the country.

Data from China's nationwide case series of 2135 pediatric patients with COVID-19 observed that more than $90 \%$ of all patients had asymptomatic, mild, or moderate cases ${ }^{3}$. In our series, most of the cases remained asymptomatic $(25 \%)$ or with mild symptoms $(51.1 \%)$. However, severe and critical cases comprised $23.4 \%$, higher than the 3-10.6\% described in the previously healthy Chinese pediatric population (according to age range $)^{3}$. Most of our patients who presented lower respiratory tract symptoms developed dyspnea and/or hypoxemia, were managed successfully in the ward with good clinical outcome. The proportion of infected children requiring intensive care management was $8.5 \%$, a higher percentage compared to that observed in the general pediatric population ${ }^{20}$. Of note, all four patients were either receiving intensive chemotherapy or severely immunosuppressed post allo-SCT. Noteworthy, laboratory abnormalities accompanying more severe forms of infection and admission to the PICU identified as surrogate markers low total lymphocyte count and high ferritin levels at onset of symptoms. One of the deaths in the post allo-SCT subgroup was probably related to an uncontrolled systemic inflammatory response that closely resembled that of secondary hemophagocytic lymphohistiocytosis (sHLH) or macrophage activation syndrome (MAS), as it has been described in other severe COVID-19 patients ${ }^{21}$. SARS-CoV-2 infection seems to trigger a cytokine storm and hyper-activation of the immune response, with consequent multiple organ damage. In this cohort 
of patients, no diagnosis of Kawasaki disease or toxic syndrome were observed as described elsewhere ${ }^{22}$.

Regarding therapy, hydroxychloroquine and antivirals (lopinavir/ritonavir, remdesivir, and oseltamivir) were used mainly in early stages ${ }^{23}$. In those with a more aggressive disease and/or hyperinflammation component, immunomodulatory treatments similar to those used in the cytokine release syndrome associated with CART therapy (i.e . tocilizumab, siltuximab, anakinra, corticosteroids) were administered ${ }^{24}$. Clear recommendations of anti-viral therapy, immunomodulation, and of an effective vaccine are not available yet, but hopefully they will be stablished soon after the ongoing basic and clinical trials progress ${ }^{25}$. According to our data, most patients will have a good outcome with supportive care. However, it may be postulated that patients receiving intensive chemotherapy, severely immunosuppressed, or GVHD may be at a higher risk of morbidity and even mortality secondary to COVID-19.

There is also the concern that during this pandemic, children with cancer may have an incremented risk of presenting a poor prognosis for their disease, due to late diagnosis and not appropriate treatment ${ }^{26-27}$. In this respect, half of the patients included in our cohort had interrupted their anticancer directed treatment for a number of days due to the concomitant infection. Larger series of cases and longer follow up information will be needed to understand its real impact. However, it is not infrequent that chemotherapy regimens have to be stopped during opportunistic infections in patients undergoing immunosuppression. Recent guidelines published from SIOP, COG, SIOP-E, SIOP-PODC, IPSO, PROS, CCI, and St Jude Global for children with cancer recommend that the standards of care for the diagnosis, treatment and supportive care should not be compromised or electively modified during the pandemic, if at all possible ${ }^{28}$. In this line and based on our experience, we recommend individualized multidisciplinary discussions whenever a COVID-19 case is identified. A number of variables including the underlying condition, stage of disease, prognosis and clinical infection related impact should be taking into consideration for interrupting or delaying anti-cancer therapy.

Limitations of this report include the small sample size, which limits the extrapolation of results to the general hemato-oncological pediatric population. Moreover, the data was collected both retrospectively and prospectively. Thus, it may be a limitation when reporting disease evolution and other time-dependent factors. Finally, as the present study is multicentric and data was collected from different hospitals around the country, a potential interindividual variability may exist when reporting the different clinical scenarios. However, since most of the physicians of the Spanish Units of PHO took part in this initiative, we believe our report represents an accurate infection prevalence among children diagnosed with cancer and chronic hematological disorders in our country.

This is one of the largest series of COVID-19 in pediatric cases with solid and hematological malignancies, benign hematologic conditions, and post allo-SCT reported during the present pandemic. Recent publications based on a low number of cases seem to suggest that immunosuppressed pediatric patients do not present an increased risk of developing severe SARS-CoV-2 infection ${ }^{616}$ Our results show that the clinical course of most patients is overall good. However, severe forms of infection can be seen in highly immunosuppressed patients or in those with chronic co-morbidities such as GVHD, or patients receiving CART cell therapy. International collaborative groups' series cases will surely be published soon in order to better establish the real impact of this infection in our vulnerable pediatric population.

\section{Conflict of Interest}

The authors disclose no conflict of interest.

This research did not receive any specific grant from funding agencies in the public, commercial, or not-forprofit sectors.

Authors' contributions: AF, JLD, SR and AML were involved in designing the study. All authors were involved with patient accrual and data collection. SPJ was the statistician of the study. AF, JLD, SPJ, SR and AML were involved with data interpretation. All authors contributed to the manuscript editing and approved the final manuscript.

AML and JLD contributed equally to this work. 


\section{Acknowledgements}

We thank all SEHOP contributing members for rapidly sharing their experience during the SARS-CoV-19 pandemic. We also would like to acknowledge all healthcare providers fighting this terrible crisis.

\section{References}

1. Li Q, Guan X, Wu P, et al. Early transmission dynamics in Wuhan, China, of novel coronavirus-infected pneumonia. N Engl J Med . 2020;382(13):1199-1207. doi:10.1056/NEJMoa2001316

2. Cevik M, Bamford C, Ho A. COVID-19 pandemic - A focused review for clinicians. Clin Microbiol Infect . 2020. doi:10.1016/j.cmi.2020.04.023

3. Dong Y, Mo X, Hu Y, et al. Epidemiological Characteristics of 2143 Pediatric Patients With 2019 Coronavirus Disease in China.Pediatrics . 2020;145(6):e20200702. doi:10.1542/peds.2020-0702

4. Coronavirus Disease 2019 in Children - United States, February 12-April 2, 2020. MMWR Morb Mortal Wkly Rep . 2020;69(14):422-426. doi:10.15585/mmwr.mm6914e4

5. Centro de Coordinación Alertas y Emergencias Sanitarias del Gobierno de España. Actualización $\mathrm{n}^{\mathrm{o}}$ 50. Enfermedad por el coronavirus (COVID-19). 20.03.2020. https://www.mscbs.gob.es/profesionales/saludPublica/ccayes/alertasActual/nCovChina/documentos/Actualizacion_50_COVID-19.pdf. Published 2020. Accessed April 27, 2020.

6. Hrusak $\mathrm{O}$, Kalina $\mathrm{T}$, Wolf $\mathrm{J}$, et al. Flash survey on severe acute respiratory syndrome coronavirus-2 infections in paediatric patients on anticancer treatment. Eur J Cancer . 2020;132:11-16. doi:10.1016/j.ejca.2020.03.021

7. Hirsch HH, Martino R, Ward KN, Boeckh M, Einsele H, Ljungman P. Fourth European conference on infections in leukaemia (ECIL-4): Guidelines for diagnosis and treatment of human respiratory syncytial virus, parainfluenza virus, metapneumovirus, rhinovirus, and coronavirus. Clin Infect Dis . 2013. doi:10.1093/cid/cis844

8. Liang W, Guan W, Chen R, et al. Cancer patients in SARS-CoV-2 infection: a nationwide analysis in China. Lancet Oncol . 2020;21(3):335-337. doi:10.1016/S1470-2045(20)30096-6

9. Dai M-Y, Liu D, Liu M, et al. Patients with Cancer Appear More Vulnerable to SARS-CoV-2: A MultiCenter Study During the COVID-19 Outbreak. SSRN Electron J . 2020. doi:10.2139/ssrn.3558017

10. Aylward, Bruce (WHO); Liang W (PRC). Report of the WHO-China Joint Mission on Coronavirus Disease 2019 (COVID-19) .; 2020.

11. von Lilienfeld-Toal M, Berger A, Christopeit M, et al. Community acquired respiratory virus infections in cancer patients - Guideline on diagnosis and management by the Infectious Diseases Working Party of the German Society for haematology and Medical Oncology. Eur J Cancer . 2016. doi:10.1016/j.ejca.2016.08.015

12. Ogimi C, Englund JA, Bradford MC, Qin X, Boeckh M, Waghmare A. Characteristics and outcomes of coronavirus infection in children: The role of viral factors and an immunocompromised state. J Pediatric Infect Dis Soc . 2019;8(1):21-28. doi:10.1093/jpids/pix093

13. Bonet RP, Romaguera EP, Poveda SV. Registro Español de Tumores Infantiles Estadísticas Cáncer infantil en España cohortes. Minist Sanidad, Serv Soc e Igual . 2019. https://www.uv.es/rnti/informes.html.

14. Centro Nacional de Epidemiología. Distribución geográfica de los casos totales y los casos de las últimas 24h (obtenidos a partir de la declaración agregada de casos del Ministerio de Sanidad). https://cnecovid.isciii.es/covid19/. Accessed May 13, 2020.

15. de Rojas T, Pérez-Martínez A, Cela E, et al. COVID-19 infection in children and adolescents with cancer in Madrid. Pediatr Blood Cancer . 2020;(April):e28397. doi:10.1002/pbc.28397 
16. D'Antiga L. Coronaviruses and immunosuppressed patients. The facts during the third epidemic. Liver Transpl . 2020:0-1. doi:10.1002/lt.25756

17. Lu X, Zhang L, Du H, et al. SARS-CoV-2 Infection in Children. N Engl J Med . 2020. doi:10.1056/NEJMc2005073

18. Chen Z, Xiong H, Li JX, et al. COVID-19 with post-chemotherapy agranulocytosis in childhood acute leukemia: a case report.Zhonghua Xue Ye Xue Za Zhi . 2020. doi:10.3760/cma.j.issn.0253-2727.2020.0004

19. André N, Rouger-Gaudichon J, Brethon B, et al. COVID-19 in pediatric oncology from French pediatric oncology and hematology centers: High risk of severe forms? Pediatr Blood Cancer . 2020;(April):e28392. doi:10.1002/pbc. 28392

20. Ludvigsson JF. Systematic review of COVID-19 in children shows milder cases and a better prognosis than adults. Acta Paediatr Int J Paediatr . 2020. doi:10.1111/apa.15270

21. Mehta P, McAuley DF, Brown M, Sanchez E, Tattersall RS, Manson JJ. COVID-19: consider cytokine storm syndromes and immunosuppression. Lancet . 2020;395(10229):1033-1034. doi:10.1016/S01406736(20)30628-0

22. Harahsheh AS, Dahdah N, Newburger JW, et al. Missed or Delayed Diagnosis of Kawasaki Disease During the 2019 Novel Coronavirus Disease (COVID-19) Pandemic. J Pediatr . April 2020:S0022-3476(20)30556-4. doi:10.1016/j.jpeds.2020.04.052

23. Sankar J, Dhochak N, Kabra SK, Lodha R. COVID-19 in Children: Clinical Approach and Management. Indian J Pediatr . 2020. doi:10.1007/s12098-020-03292-1

24. Ye Q, Wang B, Mao J. The pathogenesis and treatment of the 'Cytokine Storm" in COVID-19.' J Infect . 2020. doi:10.1016/j.jinf.2020.03.037

25. Chiotos K, Hayes M, Kimberlin DW, et al. Multicenter initial guidance on use of antivirals for children with COVID-19/SARS-CoV-2.J Pediatric Infect Dis Soc . 2020. doi:10.1093/jpids/piaa045

26. Yang C, Li C, Wang S. Clinical strategies for treating pediatric cancer during the outbreak of 2019 novel coronavirus infection.Pediatr Blood Cancer . 2020;67(5):1-2. doi:10.1002/pbc.28248

27. Balduzzi A, Brivio E, Rovelli A, et al. Lessons After the Early Management of the COVID-19 Outbreak in a Pediatric Transplant and Hemato-Oncology Center Embedded within a COVID-19 Dedicated Hospital in Lombardia, Italy. Estote Parati. (Be Ready.). SSRN Electron J . 2020. doi:10.2139/ssrn.3559560

28. Sullivan M, Bouffet E, Rodriguez-Galindo C, et al. The COVID-19 pandemic: A rapid global response for children with cancer from SIOP, COG, SIOP-E, SIOP-PODC, IPSO, PROS, CCI, and St Jude Global.Pediatr Blood Cancer . April 2020. doi:10.1002/pbc.28409

\section{Hosted file}

Table 1 PBC.docx available at https://authorea.com/users/330911/articles/457718-initialreport-on-spanish-pediatric-oncologic-hematologic-and-post-stem-cell-transplantationpatients-during-sars-cov-2-pandemic 